\title{
The Design and Simulation on DP-QPSK Demodulation Device Based on MMI
}

\author{
Weihua Liu ${ }^{1, a}$, Xiqu Chen ${ }^{1, b}$ and Chao Fang ${ }^{1, c}$ \\ ${ }^{1}$ School of Electric \& Electronic Engineering \\ Wuhan Polytechnic University, Wuhan, China

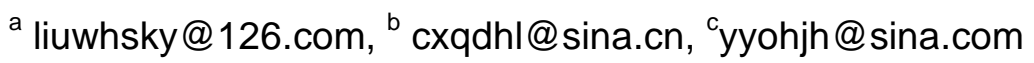

\begin{abstract}
Keywords: Planar lightwave circuits; Multimode interference; Coherent receiver.
Abstract. A DP-QPSK demodulation device what is based on Multimode Interference(MMI) cascading structure was proposal, This cascading structure does not require other additional production processes, and has good application prospect. The simulated results show that the structure can complete the polarization splitting and the phase demodulating well. In the whole C-wave band, the insertion loss is lower than $5.3 \mathrm{~dB}$, the phase error of TE and TM signal demodulating are lower than $5^{\circ}$.
\end{abstract}

\section{Introduction}

Recently, The coherent communication system what is based on DP-QPSK demodulation device has attracted wide attention ${ }^{[1,2]}$. Compared to the traditional IM-DD communication systems, the receiving terminal of the coherent communication system is complex. the signals need to be polarization split firstly. The separated QPSK signal in TE and TM polarization direction mixes with local oscillator laser, then the phase information in QPSK signal is changed into intensity information, and the intensity information was proceed by photoelectric conversion at last. The function of polarization split is completed by the polarization beam splitter(PBS), and the phase demodulating is completed by optical hybrid.

Generally, the PBS is based on crystal or polymer. But the optical hybrid is a PLC chip. If inserting the crystal into the PLC chip ${ }^{[3,4]}$, it will generate large insertion loss, complicated fabrication, and low reliability. In this paper, we proposal a DP-QPSK demodulation device what is based on MMI cascading style, the polarization separation and the phase demodulating are accomplished simultaneously. This cascading structure does not require other additional production processes, and has good application prospect.

The article designed and simulated the DP-QPSK demodulating device based on cascading MMI. The results show that the structure can complete the polarization splitting and the phase demodulating well. In the whole $\mathrm{C}$-wave band, the insertion loss is lower than $5 \mathrm{~dB}$, the phase error of TE signal demodulating is lower than $5^{\circ}$, the phase error of TM signal is also lower than $4^{\circ}$, which satisfy the need of OIF.

\section{The Design of DP-QPSK Demodulation Device}

The structure diagram of the device is shown in Fig 1 . It is composed by two $2 * 2 \mathrm{MMI}$ and two $4 * 4$ MMI. The two $2 * 2 \mathrm{MMI}$ is connected by two different widths of single mode waveguides and make up a Mach-Zehnder structure, and it is used to achieve the polarization splitting of the DP-QPSK signal ${ }^{[5]}$. The DP-QPSK signal input $2 * 2 \mathrm{MMI}$ from input terminal, then will be divided into two beams light with the same intensity, and enter into the two single mode waveguides respectively. Because the width of the two single mode waveguides are different, the generated phase differences are also different, the generated phase differences of TE and TM modules are as following:

$$
\begin{gathered}
\Delta \varphi_{1}=\left(n_{1} l-n_{1}^{\prime} l\right) 2 \pi / \lambda=\left(m_{1}+1 / 2\right) 2 \pi \\
\Delta \varphi_{2}=\left(n_{2} l-n_{2}^{\prime} l\right) 2 \pi / \lambda=m_{2} 2 \pi
\end{gathered}
$$


Where $n_{1,} n_{1}{ }^{\prime}, n_{2}$, and $n_{2}{ }^{\prime}$ are effective refractive index of TE and TM modules in the two single mode waveguides, $\lambda$ is wavelength of the signal light, $m_{1}$ and $m_{2}$ are integers. Under a certain condition of waveguides width, choosing suitable width of single mode waveguide what can make the TE and TM modes generate 180 degree phase difference, and if add the phase shift which introduced by the second $2 * 2 \mathrm{MMI}$, at the output port will come true that the light of TE constructive interference and the light phase of TM destructive interference meanwhile, and TM destructive interference and TE constructive interference in the other port. Finally, The function of polarization split is realized.

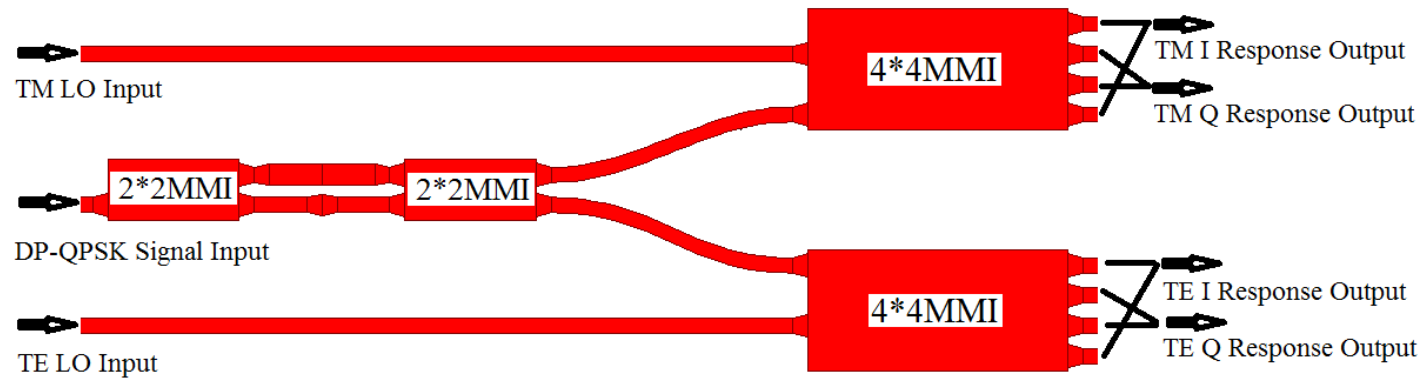

Fig. 1. Schematic diagram of DP-QPSK demodulation device

The separated TE signal light is inputted to $4 * 4 \mathrm{MMI}$, the $4 * 4 \mathrm{MMI}$ can divide light intensity into four beams. And there are $90^{\circ}, 180^{\circ}$ and $270^{\circ}$ phase differences between a beam of light and the other three beams of light. TE local oscillator laser is also separated and phase shifted by $4 * 4 \mathrm{MMI}$. The phase shifting of the QPSK signal demodulation need is just the phase difference of signal light and the local oscillator laser in the output port. Therefore, the intensities components of the outputting demodulation in the four output ports are I-, Q+, Q- and I+ respectively, I+ and I- finally input into a pair of balanced receiver and proceed by the photoelectric conversion, the Q+ and Q- will input into another balanced receiver. Similarly, the TM signal light will enter into the other $4 * 4 \mathrm{MMI}$ after beam splitting and carry out coherent with TM local oscillator laser, then will get corresponding input of TM demodulation.

In order to getting good performance index, the device is need to be designed optimally. In the paper, we used a three-dimensional (3-D) beam propagation method to simulate. The whole device is achieved on InP substrate, there is a $0.5 \mu \mathrm{m}$ thick InGaAsP in the middle, and $0.5 \mu \mathrm{m}$ thick InP up and down the middle tier. When the width is $2.5 \mu \mathrm{m}$, the polarization correlation of waveguide is minimum. So the input, output and connection waveguide of the device are single mode waveguide with $2.5 \mu \mathrm{m}$ width. The width of $2 * 2 \mathrm{MMI}$ multimode is $10 \mu \mathrm{m}$, and the width of $4 * 4 \mathrm{MMI}$ multimode is $20 \mu \mathrm{m}$, the both sides widths of the Polarization Beam Splitter are $3.6 \mu \mathrm{m}$ and $2 \mu \mathrm{m}$ respectively. In the study, the input and output ports are used tapered waveguide to connect to reduce insertion loss.

\section{Optimization and Simulation Results}

firstly we optimize the $2 * 2 \mathrm{MMI}$ and $4 * 4 \mathrm{MMI}$ in the middle of $1.55 \mu \mathrm{m}$ wave respectively, which is for getting the best performance index. Because the working principle of MMI is self-imaging based on optical waveguide, and when the width of MMI multimode is certain, MMI exists an optimum length, the imaging is the best at this moment. That is to say the loss is minimum, beam splitting is most uniform, the phase shifting is closest to the theoretical value. Therefore, we only need optimize index of the splitting ratio, and when the index is the optimal, the other indexes are also optimal. After optimizing, the optimum lengths of $2 * 2 \mathrm{MMI}$ and $4 * 4 \mathrm{MMI}$ are $435 \mu \mathrm{m}$ and $862 \mu \mathrm{m}$ respectively. The DP-QPSK demodulation is a broadband device, so we have to think about the MMI beam splitting in C-band, the results are shown in Fig2. Fig2(a) is the result of beam splitting of 2*2MMI, in the whole C-band is homogeneous; Fig2(b) is the result of beam splitting of $4 * 4 \mathrm{MMI}$, the beam splitting result is better in long wave direction than in short wave direction. Overall the beam splitting results of 
$2 * 2 \mathrm{MMI}$ are better than $4 * 4 \mathrm{MMI}$, this is because the beam splitting results of $4 * 4 \mathrm{MMI}$ are influenced by more factors.
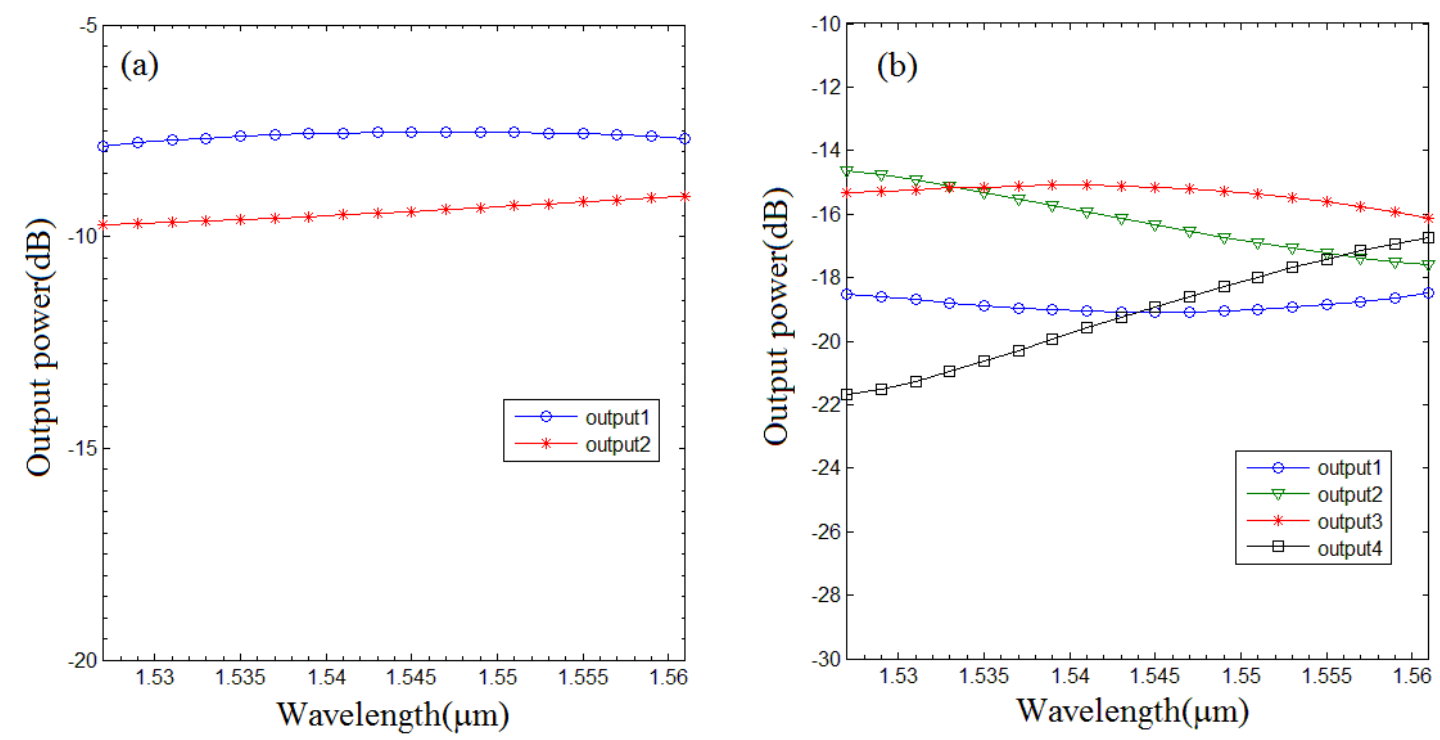

Fig. 2. The output power on the C-band for (a) $2 * 2 \mathrm{MMI}$ and(b) $4 * 4 \mathrm{MMI}$.

After getting the optimal MMI, we can optimize the Polarization Beam Splitter. The index what is to measure the device performance is extinction ratio. In the article, let's the index start with 1.55um, when the both-sides width of the Polarization Beam Splitter is certain, the best length of it is $364 \mu \mathrm{m}$, then the beam splitting results of simulating the whole C-band with the optimum size are shown in Fig.3. Where the blue line with circles is the extinction ratio of TE and the green line with triangles is the extinction ratio of TM. The biggest extinction ratios of TE and TM modes are at $1.555 \mu \mathrm{m}$ and $1.553 \mu \mathrm{m}$ respectively, it is mainly due to the subtle difference of the effective refractive index of MMI to TE and TM modes.

When we get the optimal size of DP-QPSK demodulation and checkout the function of the demodulation, the results are shown in Fig.4. The inputting signal in the input port is QPSK of the TM polarization. Most of the luminous power enters into the up $4 * 4 \mathrm{MMI}$, and carry out coherent with its local-oscillator and obtain obviously discrimination I and Q response. Because the signal enters into the below $4 * 4 \mathrm{MMI}$ is very little, it is just a beam splitter, the inputting local-oscillator is divided into four beams(4 channel).

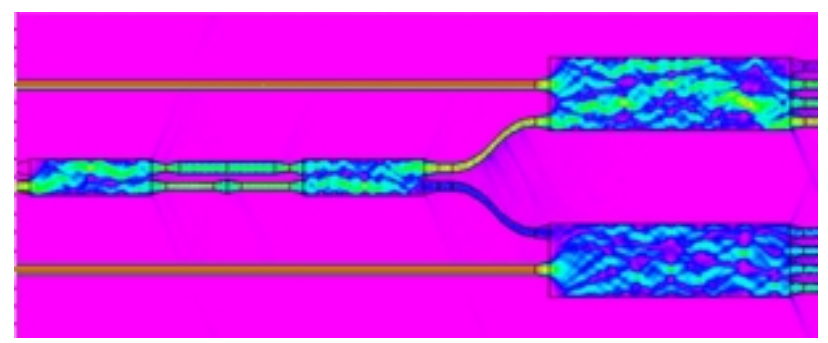

Fig. 3. Simulated light distribution of the DP-QPSK demodulation device at $1.55 \mu \mathrm{m}$ wavelength with TE polarization.

We also simulate the insertion loss and the phase deviation of DP-QPSK demodulation shown in Fig.5. Fig 5(a) is the insertion loss of the device from $3 \mathrm{~dB}$ to $5 \mathrm{~dB}$ in the whole C-band. Fig5(b) is the phase deviation. It is shown that the phase deviation is lower than $5^{\circ}$ in the whole C-band what is satisfied the specified standard of OIF 

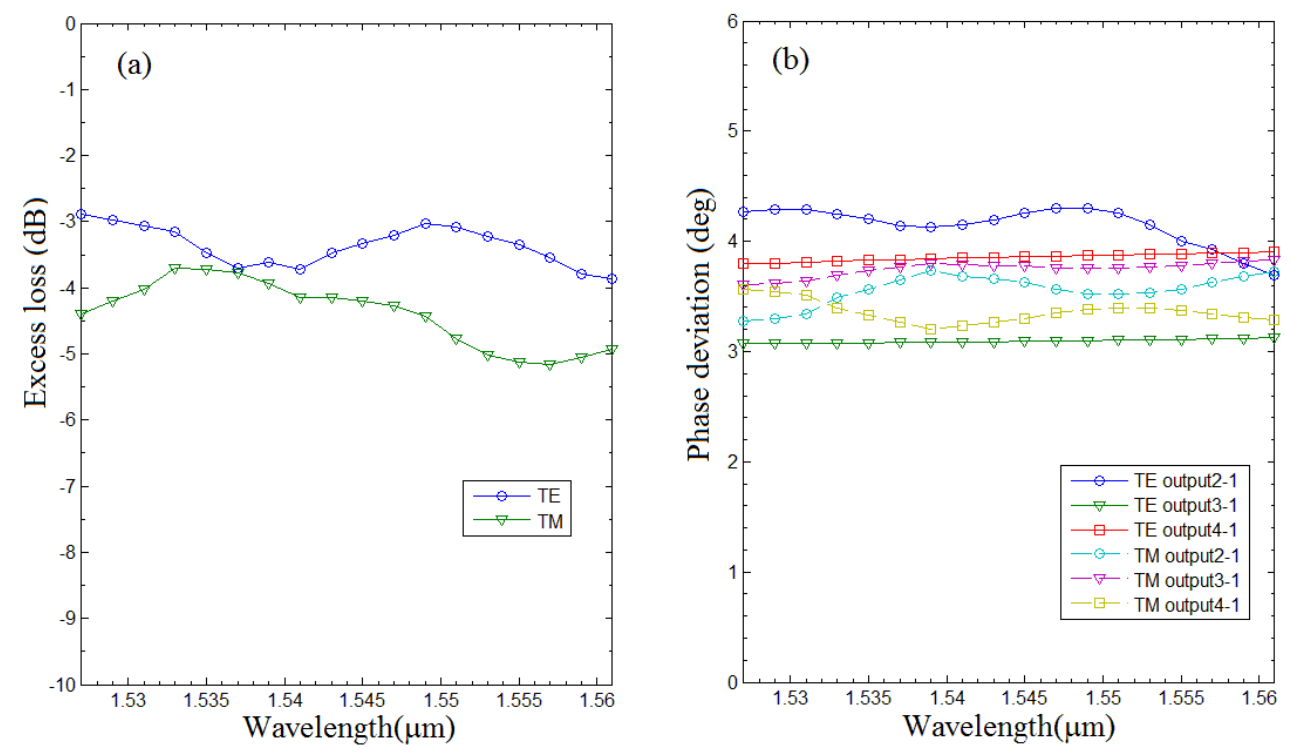

Fig. 4. Simulated the insertion loss and the phase deviation of DP-QPSK demodulation device on the C-band,(a) insertion loss,(b) phase deviation

\section{Conclusions}

The demodulation of DP-QPSK signal with cascaded MMI structure was designed and simulated, the results show that the structure can complete the functions of polarization and phase demodulation of DP-QPSK signal well. In the whole C-band the insertion ratios are lower than $5.3 \mathrm{~dB}$, the phase errors of the TE signal demodulation are lower than $5^{\circ}$, the phase errors of the TM signal demodulation are also lower than $4^{\circ}$, these are satisfied the specified standard of OIF. This cascading structure does not require other additional production processes, and has good application prospect.

\section{Acknowledgements}

This work was financially supported by the Research Project of Hubei Provincial Department of Education (B2016075).

\section{References}

[1] Ip E., Lau A. P. T., Barros D. J. F., and Kahn J. M., "Coherent detection in optical fiber systems," Optic express, vol.16, no. 753, pp.753-791, Jan. 2008.

[2] L. Zimmermann, K. Voigt, G. Winzer, K. Petermann, and C. M.Weinert, "C-band optical 90" hybrids based on Silicon-on-insulator $4 * 4$ waveguide coupler," IEEE Photonic Technology Letter, vol. 21, no.3, pp. 143-145, Feb.2009.

[3] S. H. Jeong, and K. Morito, "Novel Optical $90^{\circ}$ Hybrid Consisting of a Paired Interference Based 2 * 4 MMI Coupler, a Phase Shifter and a 2 * 2 MMI Coupler," J. Lightwave Technology, vol.28, no. 9, pp. 1323-1331, May 2010.

[4] Lingjie Wang, Yanli Zhao, Yuanzhong Xu, Tianhong Zhou, Weihua Liu, Zheng Chen, and Wen Liu, Excess Loss Reduction in Low Cost Wide Waveguide Gap Polarization Mode Converter IEEE Photonic Technology Letter, vol.25, no. 8, pp. 741-744, April 2013

[5]Y. Inoue, Y. Ohmori, M. Kawachi, S. Ando, T. Sawada, and H. Takahashi, "Polarization mode converter with polyimide half waveguide in silica-based planar lightwave circuits," IEEE Photonic Technology Letter, vol. 6, no. 5, pp. 626-628, May 1994. 